\title{
Production de litière de la mangrove du Parc National du Delta du Saloum (Sénégal)
}

\author{
Ngor NDOUR ${ }^{1 *}$, Bienvenu SAMBOU ${ }^{2}$ et Danièle Sara DIENG ${ }^{2}$ \\ ${ }^{1}$ Université Assane SECK de Ziguinchor. BP : 523 Ziguinchor, Sénégal. \\ ${ }^{2}$ Université Cheikh Anta Diop, Institut des Sciences de l'Environnement, Dakar, Sénégal. \\ "Auteur correspondant, E-mail : ngorndour@univ-zig.sn; Tél : + 2215533203
}

\section{RESUME}

Le delta du Saloum est un estuaire poissonneux du Sénégal. Les palétuviers y jouent le rôle de nurserie et de frayère à cause essentiellement de leur production de litière qui est la base de la chaîne alimentaire dans l'écosystème mangrove. Afin de monter l'importance de la litière qui tombe dans le milieu, des trappes ont été installées dans deux sites pour le captage du matériel végétal qui chute. Les productions moyennes les plus élevées sont de l'ordre de $4,6 \mathrm{~g} / \mathrm{m}^{2} / \mathrm{J}$ pour les feuilles et de $4,09 \mathrm{~g} / \mathrm{m}^{2} / \mathrm{J}$ pour les fleurs chez $R$. racemosa/harisonnii. Elles sont d'environ $4,07 \mathrm{~g} / \mathrm{m}^{2} / \mathrm{J}$ pour les feuilles et de 0,194 pour les fleurs chez $R$. mangle. Ces statistiques montrent que dans l'année, les quantités de feuilles qui chutent des palétuviers varient dans le même ordre de grandeur chez le genre Rhizophora. Par contre, les corrélations notées, entre la production de litière florale et la composition floristique des peuplements, montrent que $R$. mangle perd moins de fleurs par avortement que $R$. racemosa/harisonnii. Cette dernière contribuerait donc plus à l'enrichissement du milieu que les autres palétuviers.

(C) 2013 International Formulae Group. All rights reserved.

Most clés : palétuviers, production, litière, Rhizophora, feuilles, fleurs.

\section{INTRODUCTION}

Le Parc National du Delta du Saloum (PNDS) se trouve dans la zone côtière du Sénégal. Il abrite la mangrove septentrionale la plus importante de l'Afrique de l'Ouest. Le relief y est globalement plat. La mangrove y est caractérisée par la famille des Rhizophoraceae représentée par trois espèces : Rhizophora racemosa Meyer, $R$. harisonnii Leechm et $R$. mangle L. Les Avicenniaceae sont représentées par Avicennia germinans L. À ces deux familles qui constituent l'essentiel des palétuviers, s'ajoute celle des Combretaceae représentée par Conocarpus erectus L. et Laguncularia racemosa (L.) C.F. Gaertn. La mangrove du PNDS souffre ces dernières années de la rupture de la flèche de Sangomar, de la sécheresse et de l'action destructrice de l'homme (Soumaré, 1992; Diaw, 1997 ; Thomas et al., 1997). La baisse 
des précipitations a accru la concentration en sel des eaux estuariennes du Delta, ce qui participe à la dégradation de la mangrove. Cette dégradation s'explique, entre autres, par la salinité excessive des eaux, leur $\mathrm{pH}$ très élevé, la présence de sols sulfatés acides, l'ensoleillement etc. (Marius, 1984; Blasco, 1984 ; Diouf, 1996 ; Ndour, 2005). Malgré ces diverses contraintes, les palétuviers contribuent à l'enrichissement du milieu par les quantités de litière qu'ils libèrent annuellement au sein de l'écosystème. Cette litière est transformée en matière minérale par les microorganismes lors du recyclage de la matière organique (FAO, 1994 ; Rajendran et Kathiresan, 2007; Ndour et al., 2012). Constitué majoritairement de feuilles, ce matériel végétal contribue à l'amélioration du taux de recouvrement du milieu pendant 12 mois, du fait que les palétuviers sont des espèces sempervirentes. L'amélioration des conditions écologiques, par les palétuviers, favorise le développement des ressources halieutiques. Ces dernières sont source de devise et de moyens d'existence pour les populations côtières. Il serait donc important d'étudier la production de litière du parc qui constitue l'entité la mieux conservée de la Réserve de Biosphère du Delta du Saloum (RBDS). A cet effet, la présente étude cherche à fournir des informations sur les quantités de litière issues des palétuviers. La connaissance de ces informations devrait permettre de mieux reconsidérer la mangrove et son espace vital.

\section{MATERIEL ET METHODES}

Le PNDS, qui constitue la zone d'étude, est limité à l'Est par le bolon Missirah, le bolon qui mène à Bakadadji et la Forêt de Fathala, à l'Ouest par l'Océan Atlantique, au Nord par le Bandiala, et au Sud par la Gambienne (Figure 1). Le climat y est l'un des facteurs principaux qui déterminent l'évolution des groupements littoraux (mangrove et végétation des cordons sableux). Considéré comme sub-guinéen par Trochain (1940), ce climat a été qualifié de soudanosahélien par Marius (1984). Ces différentes classifications sont liées principalement au glissement des isohyètes vers le Sud caractérisant une baisse de la pluviométrie dans la zone d'étude. Néanmoins, le PNDS abrite encore la plus belle et la plus haute mangrove du Delta du Saloum.

Deux sites d'étude ont été choisis dans le PNDS notamment à Koumbeing et à Bakadadji. Dans la zone de Koumbeing deux parcelles de suivi sont installées. L'un dans le peuplement de Rhizophora racemosal harisonnii de taille haute $(14 \mathrm{~m})$ qui présente un taux de recouvrement de $70 \%$. Peuplement assez étendu, une parcelle de suivi de $20 \mathrm{~m} \mathrm{x}$ $20 \mathrm{~m}$ y a été installée. Dans le peuplement de R. mangle de hauteur moyenne $6 \mathrm{~m}$ et de $40 \%$ de taux de recouvrement, une parcelle de suivi de $5 \mathrm{~m}$ x $5 \mathrm{~m}$ a été installée. Cette différence de taille des parcelles de suivi tient compte de la taille des peuplements et de leur représentativité en termes d'occupation de l'espace. Ainsi, la rareté et la faible étendue des peuplements de Rhizophora mangle font qu'une seule parcelle a été installée à Koumbeing. Dans la zone de Bakadadji, une parcelle de suivi carrée de $5 \mathrm{~m}$ de côté est installée dans un peuplement de $R$. racemosa/harisonnii de hauteur moyenne de 5 m et d'environ $90 \%$ de taux de recouvrement. Dans cette même zone, une parcelle de suivi carrée de $20 \mathrm{~m}$ de côté est aussi installée dans un peuplement de $R$. racemosa/harionnii de hauteur moyenne $13 \mathrm{~m}$ et d'environ $64 \%$ de taux de recouvrement. La raison justificative de la différence de taille des parcelles de suivi reste la même pour les deux sites.

Lors de la collecte des données dans les parcelles de suivi, la majorité des espèces a été identifiée à l'aide de la clé de 
détermination de Bérhaut (1967) et des critères de distinction évoqués par Marius (1984) et Bertrand (1993). Les critères de détermination des espèces de palétuviers donnés par ces divers auteurs n'ont pas permis de distinguer Rhizophora racemosa et $R$. harisonnii. Le suivi des inflorescences des espèces Rhizophora racemosa/harisonnii a permis de constater une évolution du stade 4 boutons floraux au stade 68 , ce qui n'a pas été évoqué dans aucune des clés de ces auteurs. De surcroît, sur une même tige de Rhizophora racemosa/harisonnii, on a retrouvé au même moment le stade 4 fleurs ouvertes indiquant la fin de la division de l'inflorescence, le stade 32 et 68. Etant donné que chez $R$. racemosa le nombre de fleurs doit être compris entre 4 et 16 fleurs et entre 16 et 32 fleurs chez $R$. harisonnii, les faits observés sur le terrain ont rendu impossible la distinction entre ces deux espèces. La collecte de litière a porté donc sur $R$. mangle et Rhizophora racemosa/harisonnii. Elle a nécessité l'installation de parcelles de suivi dans la mangrove de taille haute $(>7 \mathrm{~m})$ à moyenne $(5$ à $7 \mathrm{~m}$ ). Quatre parcelles de suivi sont installées dans le PNDS et leur taux de recouvrement déterminé par la projection de la couronne des arbres au sol. Leur dimension est variable en fonction de la taille des peuplements ciblés; alors que leur distribution dans l'espace a tenu compte de la taille des peuplements et de leur composition floristique. La parcelle de suivi est de $20 \mathrm{~m} \mathrm{x}$ $20 \mathrm{~m}$ dans les formations hautes. Celle-ci est divisée en quatre placettes carrées de $10 \mathrm{~m}$ de côté. En prenant comme repère un des angles de la parcelle de suivi et en progressant dans le sens des aiguilles d'une montre, trois trappes ont été placées au milieu de trois des placettes successives. Au niveau des parcelles de suivi de $5 \mathrm{~m}$ x $5 \mathrm{~m}$, installées dans les peuplements de taille moyenne, une trappe est placée au milieu. La litière des palétuviers a été collectée dans ces parcelles de suivi à l'aide de trappes. La trappe est l'outil de collecte de la litière qui a été conçu en s'inspirant du modèle de Brown (1984). C'est un outil en forme de panier de $50 \mathrm{~cm}$ x $50 \mathrm{~cm}$ d'ouverture x $25 \mathrm{~cm}$ de fond. Ses parois (fond et flancs) sont recouvertes d'une moustiquaire en plastique à maille de $1 \mathrm{~mm}$ x $1 \mathrm{~mm}$. Ce dispositif, placé au-dessus de la zone de balancement des marées, permet de limiter les rétentions de l'humidité des feuilles accumulées dans son fond (Figure 2). Il est suspendu à quatre piquets ou aux branches de l'individu le plus proche. La litière est piégée au fond de la trappe (Figure 3). Pour éviter sa décomposition dans la trappe et des pertes de matière organique, elle est collectée tous les deux mois à l'aide de sachets en plastique.

La localisation, la date et le numéro des trappes sont notés sur les sachets en plastique pour éviter les confusions. La litière collectée est séchée à l'étuve à $70{ }^{\circ} \mathrm{C}$ pendant 72 heures pour obtenir une matière organique anhydre et de masse constante. Elle est ensuite triée pour séparer les différents éléments (feuilles, inflorescences, propagules, brindilles, écorces...) des autres éléments tels que les restes de poissons, d'insectes, les plumes d'oiseaux... Les éléments anhydres, issus des végétaux, sont pesés à l'aide d'une balance électronique de précision 1 gramme pour la détermination de leur biomasse. Les données obtenues ont été traitées à l'aide du progiciel Excel. 


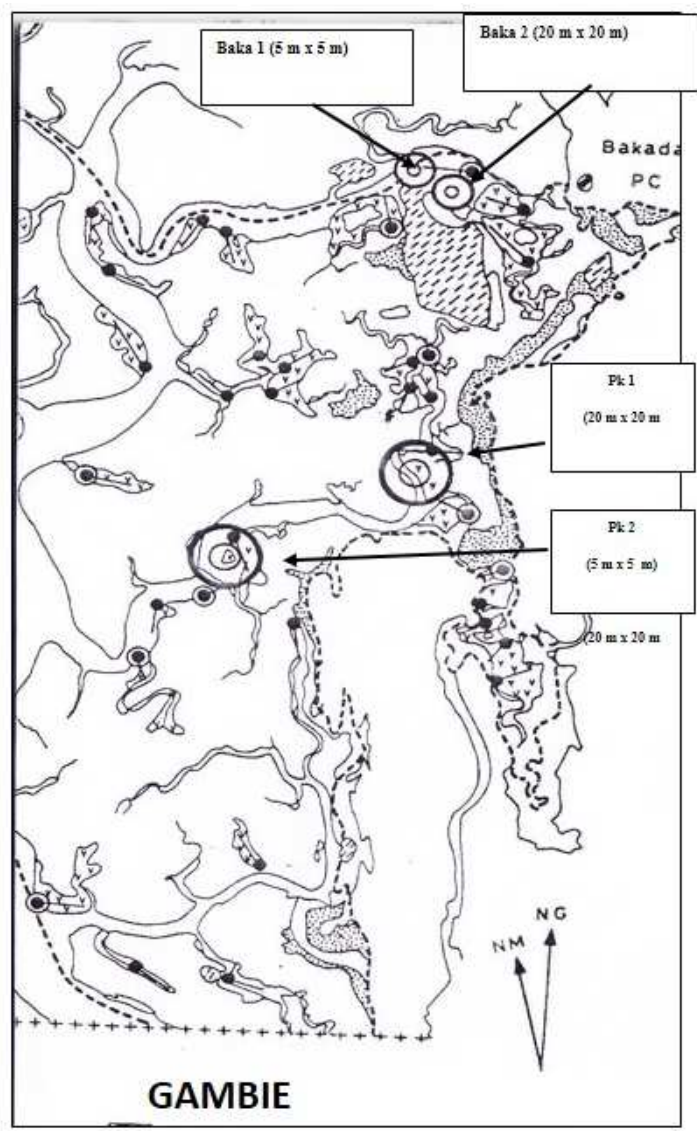

La RBDS se trouve au centre ouest du Sénégal.

Baka1 : désigne la parcelle de suivi de $5 \mathrm{mx} 5 \mathrm{~m}$ de la zone de Bakadadji

Baka2 : désigne la parcelle de suivi de $20 \mathrm{~m}$ x $20 \mathrm{~m}$ de la zone de Bakadadji.

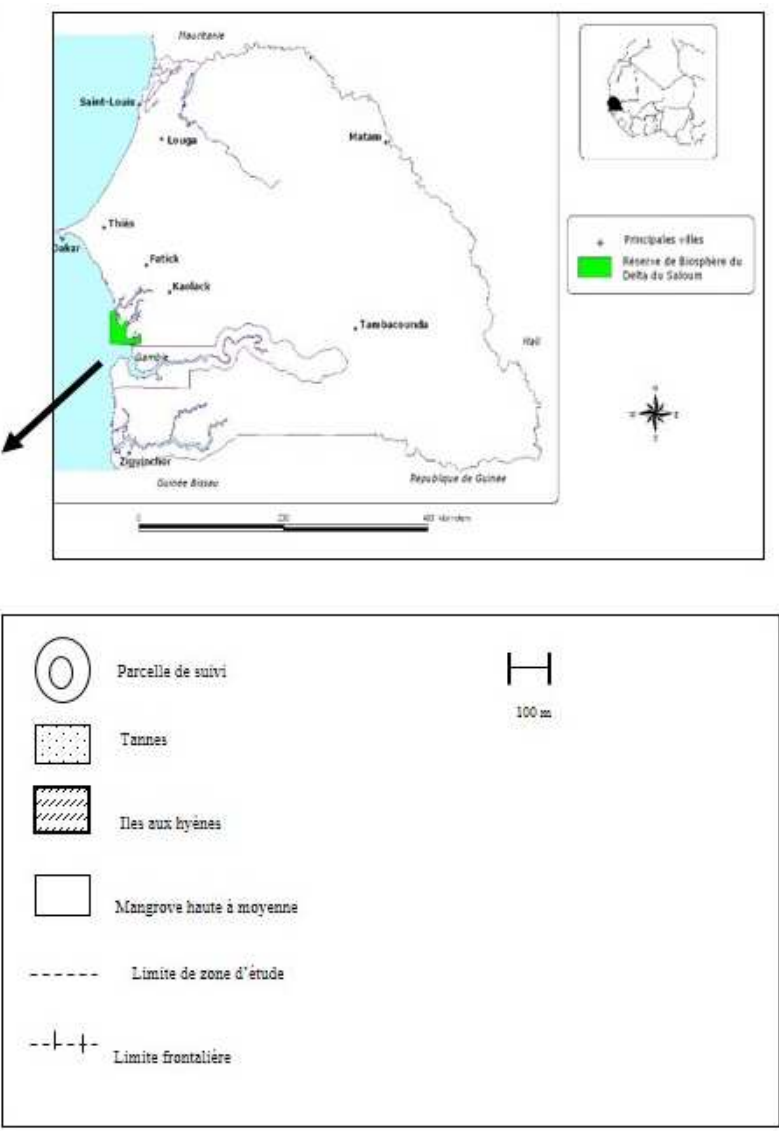

PK1 : désigne la parcelle de 20 m x 20 m de Koumbeing PK2 : désigne la parcelle de $5 \mathrm{~m}$ x $5 \mathrm{~m}$ de Koumbeing Les parcelles de suivi se trouvent dans le PNDS qui est une partie de la RBDS.

Figure 1 : localisation des sites dans la Réserve de Biosphère du Delta du Saloum.

\section{RESULTATS}

Les résultats de l'étude s'articulent autour de la production de litière par parcelle de suivi en tenant compte de la taille des peuplements et de leur composition floristique. Pour chaque parcelle, les données sont analysées et les résultats comparées entre eux en fonction de la composition floristique et de la taille des peuplements d'une part et, en fonction de la composition floristique, la taille et le taux de recouvrement du peuplement d'autre part.

\section{Production de litière de la première parcelle de suivi $\mathbf{n}^{\circ} \mathbf{1}$}

Dans la zone de Koumbeing, la quantité de litière produite, par la parcelle de suivi, est variable en fonction de la trappe et de la période de collecte (Figures $4 a, 4 b$ et 4c). 
Au niveau de la trappe 1 (Figure 4a), les productions les plus importantes concernent les feuilles et les fleurs de $R$. racemosa/harisonnii. Un maximum de production foliaire est enregistré au mois de novembre 2000 avec des quantités comprises entre 5 et $5,5 \mathrm{~g} / \mathrm{m}^{2} / \mathrm{J}$. Les pertes de fleurs sont remarquables avec deux pics voisins de 2 $\mathrm{g} / \mathrm{m}^{2} / \mathrm{J}$ enregistrés au mois de juillet pendant la durée d'exposition de la trappe. La production de propagules est légèrement supérieure à 1 $\mathrm{g} / \mathrm{m}^{2} / \mathrm{J}$ en mai 2000. Cette période coïncide avec le niveau de production de feuilles le plus bas $\left(<1,5 \mathrm{~g} / \mathrm{m}^{2} / \mathrm{J}\right)$. La quantité de bois libérée par la mangrove est inférieure à 1 $\mathrm{g} / \mathrm{m}^{2} / \mathrm{J}$. Elle est plus importante durant le mois de septembre qui coïncide avec la saison des pluies. Les vents forts, qui accompagnent les pluies au cours de cette période, accroissent la chute de bois mort dans le milieu.

Au niveau de la trappe 2 (Figure 4b), les productions de feuilles, propagules et fleurs sont les plus importantes. Le pic de production des feuilles est supérieur à $4 \mathrm{~g} / \mathrm{m}^{2} / \mathrm{J}$ en novembre 2000 . Le niveau de production de feuilles le plus bas (inférieur à $1,5 \mathrm{~g} / \mathrm{m}^{2} / \mathrm{J}$ ) est enregistré au mois de mai en 2000 et en 2001. La production de propagules est importante en mai 2000 et dans une moindre mesure en mai 2001. Les quantités maximales de propagules varient entre 0,5 et $3 \mathrm{~g} / \mathrm{m}^{2} / \mathrm{J}$. Les pertes de fleurs ont atteint à chaque fois leur pic maximum au mois de juillet pour des valeurs comprises entre 3 et $4,5 \mathrm{~g} / \mathrm{m}^{2} / \mathrm{J}$. Par ailleurs, il apparaît au niveau de la Figure $4 \mathrm{~b}$ que la floraison de l'espèce $R$. racemosa/harisonnii reprend avant la phase de maturation des propagules issues de la génération de fleurs précédentes. Ainsi, des fleurs et/ou des propagules sont observées à tout moment sur les individus de l'espèce. La quantité de bois libérée, par chute de débris entre les mois de juillet et septembre 2001, est d'environ $3 \mathrm{~g} / \mathrm{m}^{2} / \mathrm{J}$.

Au niveau de la trappe 3 (Figure 4c), les productions les plus importantes restent celles des feuilles, des propagules et des fleurs. La variation de la quantité de feuilles produites présente un pic en novembre 2000. La production de feuilles au cours de l'exposition de la trappe varie entre 3 et 5 $\mathrm{g} / \mathrm{m}^{2} / \mathrm{J}$. Celle des propagules devient relativement importante en juillet 2001. Les quantités maximales obtenues varient entre 0,5 et $3 \mathrm{~g} / \mathrm{m}^{2} / \mathrm{J}$. Les pertes de fleurs de 2 à 3,5 $\mathrm{g} / \mathrm{m}^{2} / \mathrm{J}$ sont maximales entre les mois de mai et septembre de l'année 2001. Ces quantités montrent que le taux d'avortement des fleurs de l'espèce $R$. racemosa/harionnii est très important. La production de bois est faible (1 $\mathrm{g} / \mathrm{m}^{2} / \mathrm{J}$ ) entre les mois de juillet et septembre de la même année.

La production de litière du peuplement de $R$. racemosa/harisonnii a été quantifiée en moyenne pour toutes les trappes. Cette mesure a permis de voir que la quantité moyenne de feuilles produites est de $2,80 \mathrm{~g} / \mathrm{m}^{2} / \mathrm{J}$, celle des fleurs de $1,01 \mathrm{~g} / \mathrm{m}^{2} / \mathrm{J}$, celle des propagules de $0,36 \mathrm{~g} / \mathrm{m}^{2} / \mathrm{J}$, celle du bois de $0,30 \mathrm{~g} / \mathrm{m}^{2} / \mathrm{J}$ et celle des écorces de $0,02 \mathrm{~g} / \mathrm{m}^{2} / \mathrm{J}$ dans la mangrove haute.

\section{Production de litière de la parcelle de suivi $\mathbf{n}^{\circ} 2$}

Dans la zone de Bakadadji, la quantité de litière produite au niveau de la parcelle est variable dans le temps (Figure 5).

Les productions les plus importantes concernent les feuilles. Trois pics de production sont enregistrés aux mois de juillet et de novembre 2000 ainsi qu'en juillet 2001 avec des quantités comprises entre 2 et 4 $\mathrm{g} / \mathrm{m}^{2} / \mathrm{J}$. La production de propagules est relativement faible. Elle a été notée en janvier et en mai 2001 avec des productions nettement inférieures à $1 \mathrm{~g} / \mathrm{m}^{2} / \mathrm{J}$. Les pertes de fleurs sont relativement faibles en termes de quantité. Malgré cette situation, la production en propagules est faible, ce qui pourrait être liée à une faible productivité florifère entre autres facteurs explicatifs.

En plus de l'analyse de la variation de la production de litière par trappe, la production moyenne journalière de litière a été quantifiée. 
Ainsi, la quantité de feuilles produite est en moyenne de $2,04 \mathrm{~g} / \mathrm{m}^{2} / \mathrm{J}$ ' celle des fleurs de 0,06 , celle des propagules de 0,08 et celle du bois de 0,02 .

\section{Production litière de la parcelle de suivi $n^{\circ} 3$}

La quantité de litière produite varie en fonction de la période de collecte de la litière (Figure 6). Les productions les plus importantes concernent les feuilles et les fleurs. La production maximale des feuilles est enregistrée au mois de novembre 2000 pour une valeur de $4 \mathrm{~g} / \mathrm{m}^{2} / \mathrm{J}$. La production la plus basse est nettement inférieure à 0,5 $\mathrm{g} / \mathrm{m}^{2} / \mathrm{J}$ et correspond au mois de mai 2001. La production de propagules est quasi nulle au cours de l'étude. Les pertes de fleurs sont considérables au niveau de cette parcelle. Deux pics de production variant entre 3 et 4 $\mathrm{g} / \mathrm{m}^{2} \mathrm{~J}^{-1}$ sont observés au mois de juillet 2000 et 2001. Il est à constater que les quantités de fleurs perdues sont supérieures à celles qui sont enregistrées pour les feuilles entre mai et septembre 2000 de même qu'au cours de la période mai - juillet 2001. Les pertes de fleurs les plus faibles correspondent à des périodes de reprise de la floraison chez Rhizophora racemosa/harisonnii.

Suite à l'analyse de la variation de la quantité de litière en fonction du temps, la production litière moyenne de la parcelle a été quantifiée. Ainsi, la quantité de feuilles produites est en moyenne de $2,06 \mathrm{~g} / \mathrm{m}^{2} / \mathrm{J}$, celle des fleurs de 1,62 , celle du bois de 0,06 et celle des écorces de $0,04 \mathrm{~g} / \mathrm{m}^{2} / \mathrm{J}$.

\section{Production de litière de la parcelle de suivi $n^{\circ} 4$}

Dans la zone de Bakadadji, la quantité de litière produite au niveau de cette parcelle est variable d'une trappe à l'autre (Figures 7a, $7 b$ et $7 c)$.

$\mathrm{Au}$ niveau de la Figure 7a, les productions les plus importantes concernent les feuilles et les fleurs de Rhizophora racemosa/harisonnii. Deux pics sont enregistrés aux mois de septembre 2000 et de janvier 2001 avec des valeurs comprises entre 2,5 et $4 \mathrm{~g} / \mathrm{m}^{2} / \mathrm{J}$. Le niveau de production de feuilles le plus bas est noté en juillet 2001 . Les pertes de fleurs sont importantes avec une production de $3,5 \mathrm{~g} / \mathrm{m}^{2} / \mathrm{J}$ au mois de septembre 2000. La quantité de propagules produites est inférieure à $0,5 \mathrm{~g} / \mathrm{m}^{2} / \mathrm{J}$. Leur production maximale de fleurs comprise entre 2 et $3,5 \mathrm{~g} / \mathrm{m}^{2} / \mathrm{J}$ est en phase avec la production maximale de feuilles en 2000 et 2001 pendant le mois de septembre. Le phénomène inverse s'observe entre le mois de janvier et celui de mai en 2001. Les pertes de fleurs sont faibles de janvier à mai 2001. Les quantités de bois sont inférieures à $0,5 \mathrm{~g} / \mathrm{m}^{2} / \mathrm{J}$ et apparaissent pour l'essentiel pendant les mois de juillet, septembre et novembre.

$\mathrm{Au}$ niveau de la Figure 7b, les productions de feuilles, fleurs et propagules sont les plus importantes. Celle des feuilles montre deux pics compris entre 3 et $5 \mathrm{~g} / \mathrm{m}^{2} / \mathrm{J}$ en novembre 2000 et en juillet 2001. La production de propagules est peu importante en mai 2000 et relativement importante entre juillet et septembre 2001. Les quantités maximales produites varient entre 0,5 et 2 $\mathrm{g} / \mathrm{m}^{2} / \mathrm{J}$. Les pertes maximales de fleurs sont observées au mois de juillet pour des valeurs comprises entre 2 et $4 \mathrm{~g} / \mathrm{m}^{2} / \mathrm{J}$. Elles deviennent faibles à nulles de novembre à mars 2001 . Cette période correspond à la reprise de la floraison de Rhizophora racemosa/harisonnii.

$\mathrm{Au}$ niveau de la Figure 7c, les productions les plus importantes restent celles des feuilles et des fleurs. Cette figure montre un pic de la production de feuilles supérieur à $4 \mathrm{~g} / \mathrm{m}^{2} / \mathrm{J}$ en novembre 2000. Une faible production de propagules a été notée en juillet 2001. Les pertes de fleurs sont maximales aux mois de juillet 2000 et 2001 où elles dépassent les quantités de feuilles produites au même moment. Le taux d'avortement des fleurs de l'espèce $R$. racemosa/harisonnii est très important au niveau de cette parcelle. Par ailleurs, la production litière toutes trappes confondues a été quantifiée. Ainsi, la quantité de feuilles produite est de $2,35 \mathrm{~g} / \mathrm{m}^{2} / \mathrm{J}$, celle 
des fleurs de 1,10 , celle des propagules de 0,11 , celle du bois de 0,06 et celle des écorces de 0,01 .

\section{Analyse comparative des parcelles de suivi}

Cette analyse est faite en fonction de la composition floristique et de la taille des peuplements ciblés. Les quantités de litière produites sont comparées entre la parcelle de suivi 1 et la parcelle 4 qui appartiennent à la même strate verticale et composition floristique. La première comparaison montre une évolution semblable des quantités de litière quels que soient les éléments constitutifs de celle-ci. Il apparaît qu'il n'y a pas de différence de production litière entre les parcelles 1,3 et 4 quelle que soit la différence de taille des peuplements de $R$. racemosa /harisonnii. La deuxième comparaison, entre les parcelles 2 à $R$. mangle et la parcelle 3 à $R$. racemosa /harisonnii, se fonde uniquement sur l'appartenance à la même strate verticale de leur peuplement. Elle montre que le taux d'avortement des fleurs de $R$. racemosa /harisonnii est plus élevé que celui de $R$. mangle. Cette situation explique sans doute la faible production en propagules de $R$. racemosa/harisonnii. Quant à $R$. mangle qui ne connaît pas cette situation, sa production de semences est toujours plus importante. La troisième comparaison intègre le taux de recouvrement des parcelles qui varie de $40 \%$ à $90 \%$. Elle montre que la quantité maximale de feuilles produite est indépendante du taux de recouvrement et de la taille des peuplements (Figure 8). Cette situation pourrait s'expliquer par le feuillage des palétuviers qui ne se renouvelle pas selon un rythme saisonnier et reste vert toute l'année. La quantité de fleurs avortées, quant à elle, dépend de la composition floristique du peuplement. Ainsi, les pertes de fleurs sont minimes chez $R$. mangle comparée aux autres palétuviers du même genre.

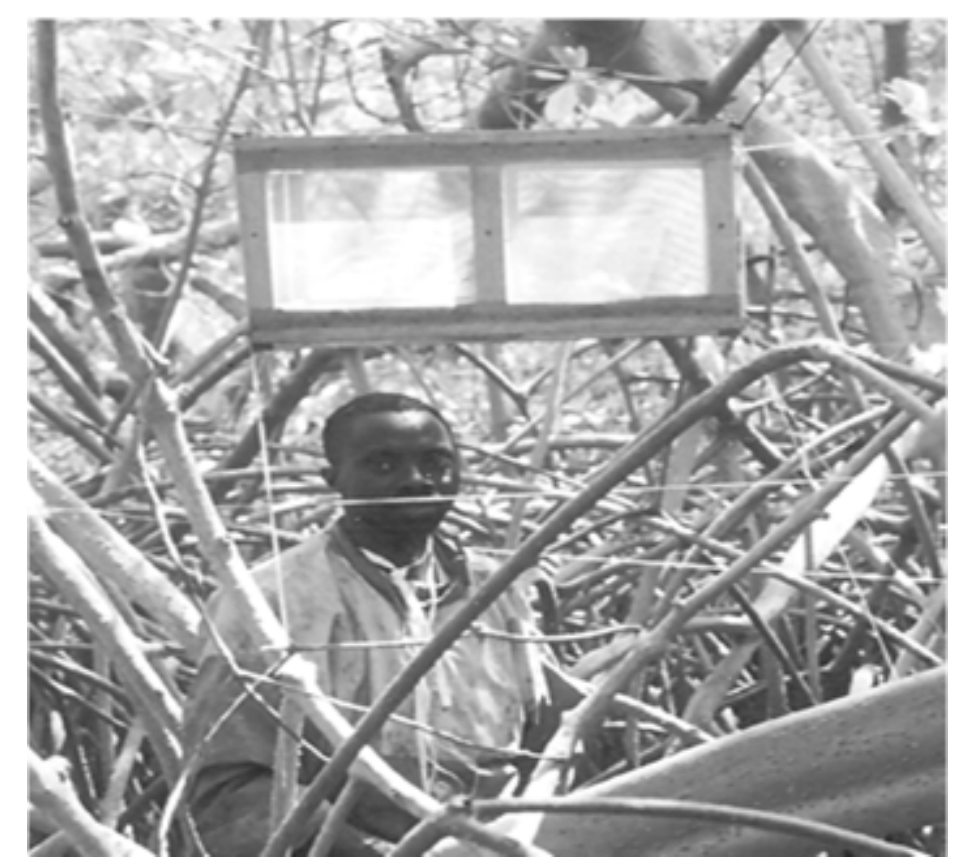

Figure 2 : Emplacement d'une trappe dans les peuplements de palétuviers.

La trappe est suspendue au-dessus de la zone de balancement des vagues pour éviter toute imbibition de la litière à marée haute. Dans les peuplements de $R$. racemosa/harosonnii, elle peut se trouver à plus de $2 \mathrm{~m}$ du sol. 
N. NDOUR et al. / Int. J. Biol. Chem. Sci. 7(6): 2349-2369, 2013

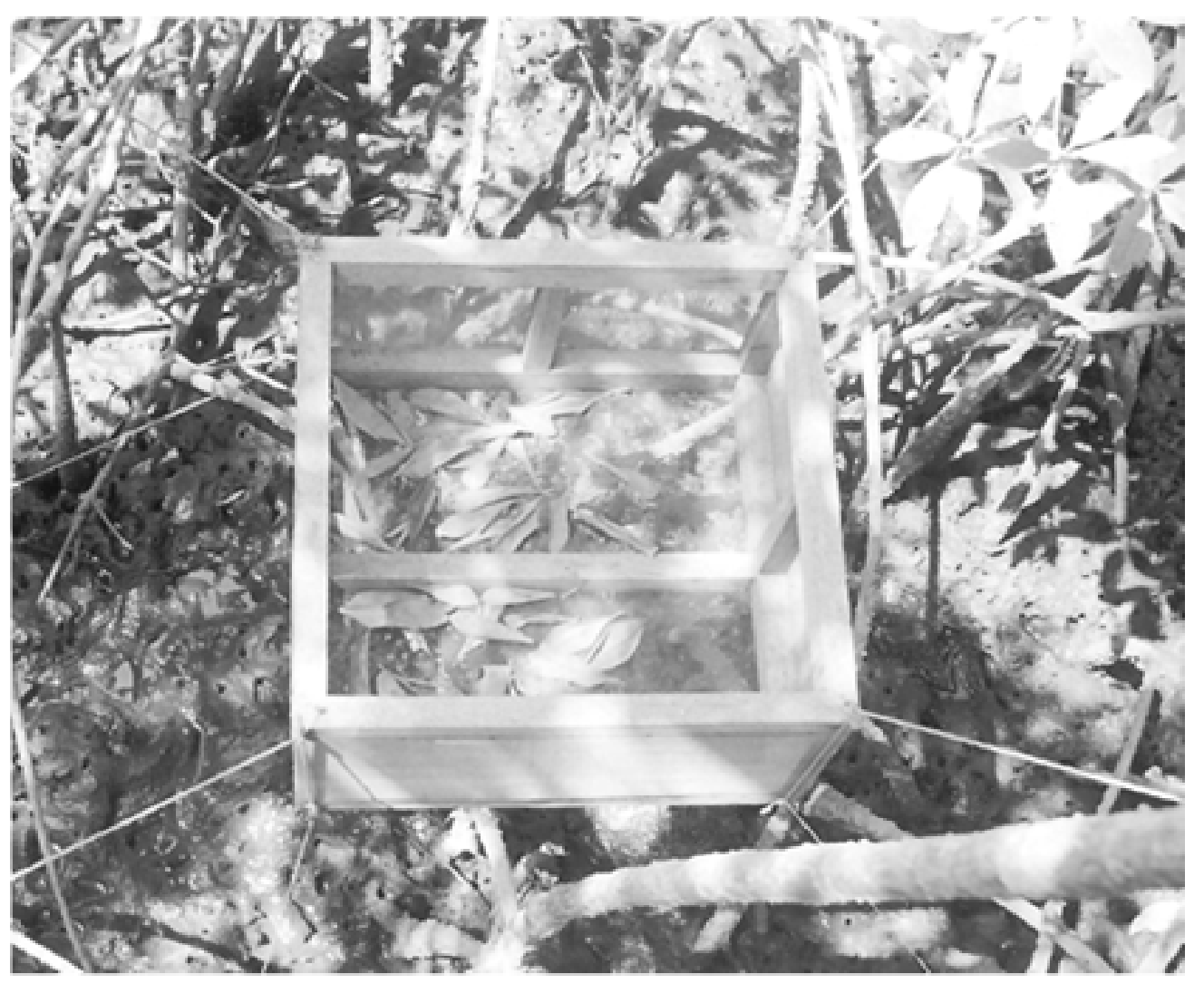

Figure 3 : Captage de la litière dans une trappe. Le matériel végétal qui tombe dans la trappe est récupéré périodiquement pour des analyses au laboratoire. 
N. NDOUR et al. / Int. J. Biol. Chem. Sci. 7(6): 2349-2369, 2013

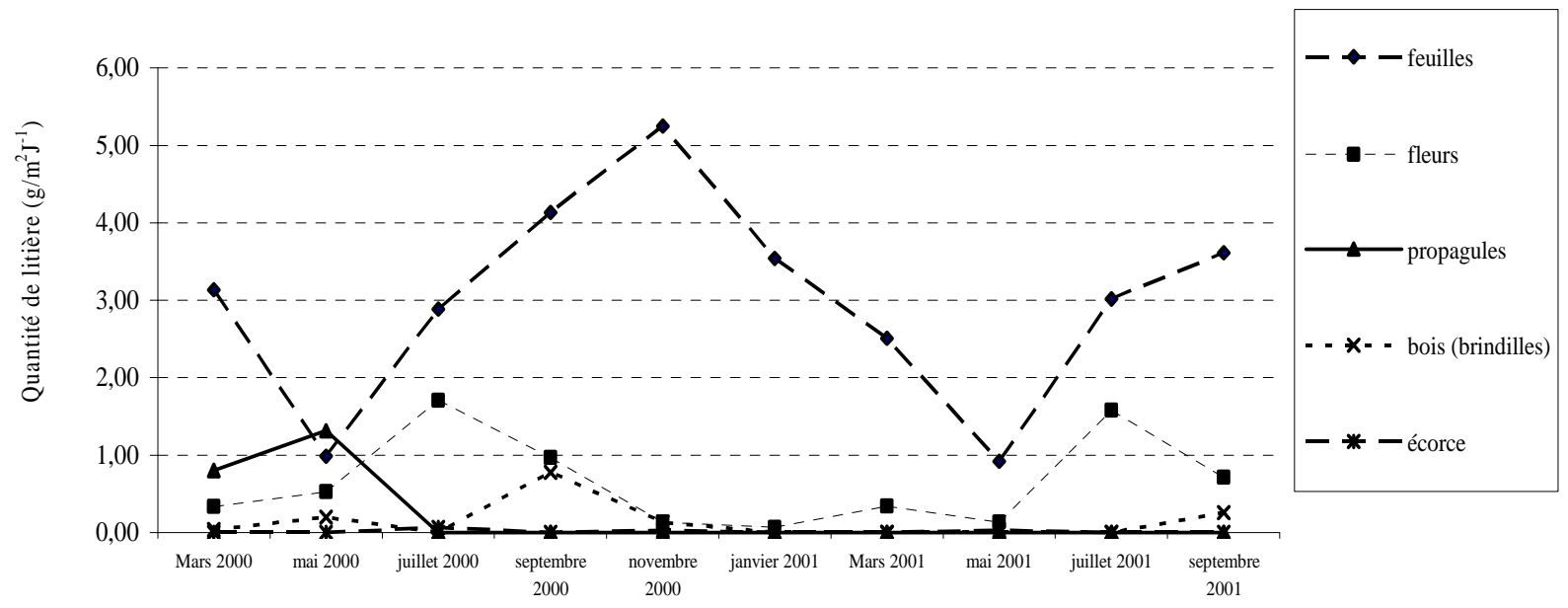

Figure 4a : Variation de la quantité de litière produite par Rhizophora racemosa/harisonnii dans la trappe 1. 


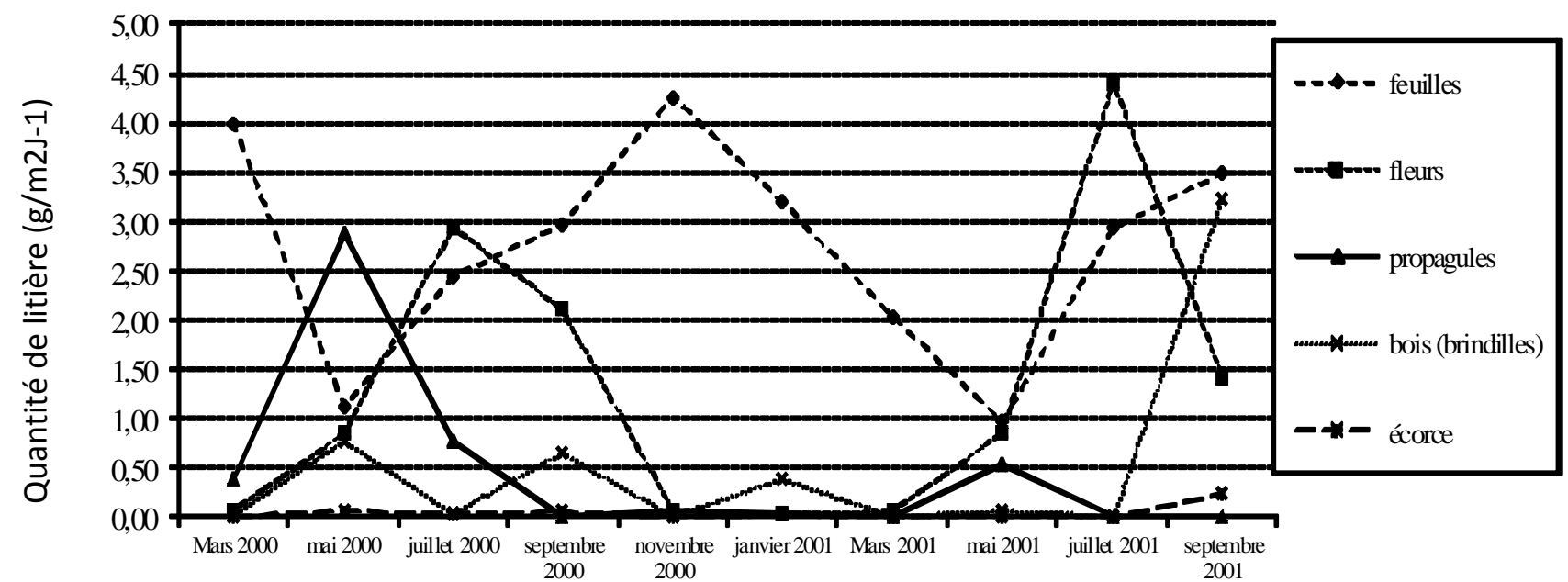

Figure 4b : Variation de la quantité de litière produite par Rhizophora racemosa/harinonnii dans la trappe 2. 
N. NDOUR et al. / Int. J. Biol. Chem. Sci. 7(6): 2349-2369, 2013

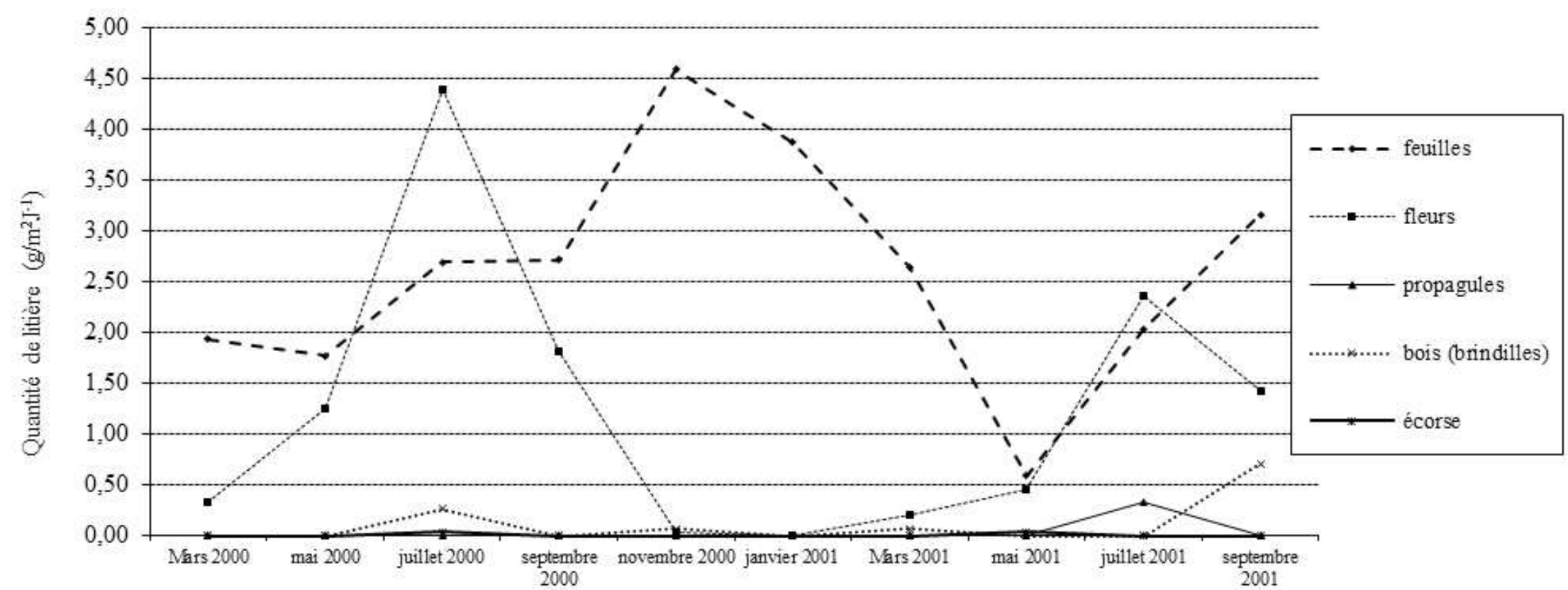

Figure 4c : Variation de la quantité de litière produite par le peuplement de Rhizophora racemosa/harisonnii dans la trappe 3. 
N. NDOUR et al. / Int. J. Biol. Chem. Sci. 7(6): 2349-2369, 2013

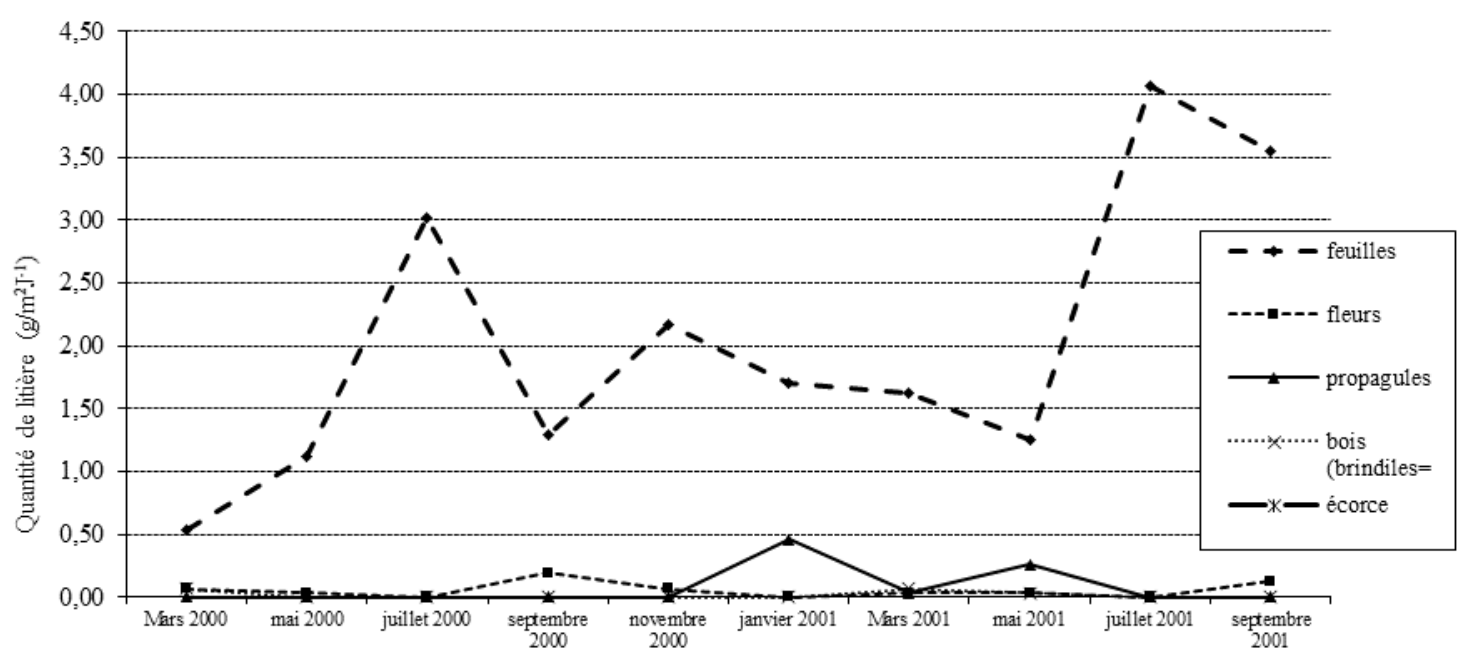

Figure 5 : Variation de la quantité litière produite par Rhizophora mangle 
N. NDOUR et al. / Int. J. Biol. Chem. Sci. 7(6): 2349-2369, 2013

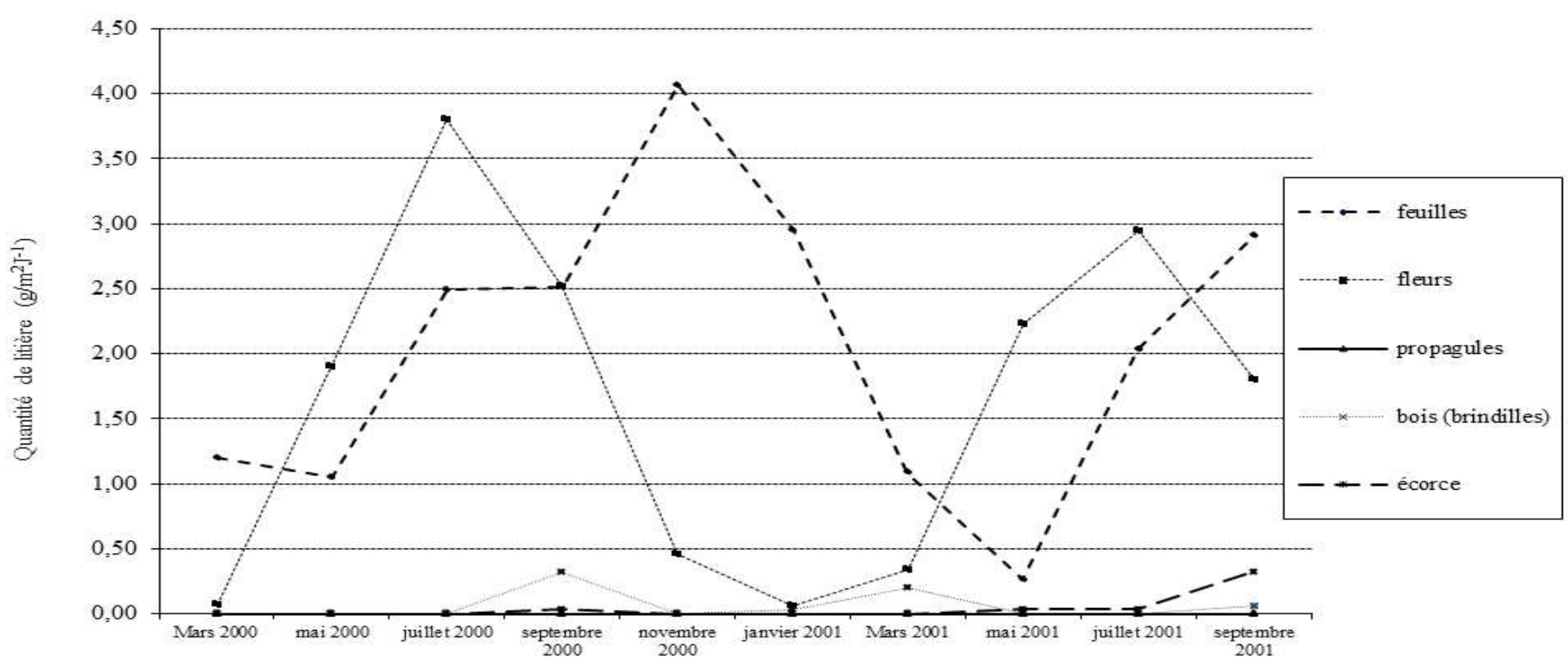

Figure 6: Variation de la quantité de litière produite par le peuplement de Rhizophora racemosa/harisonnii. 


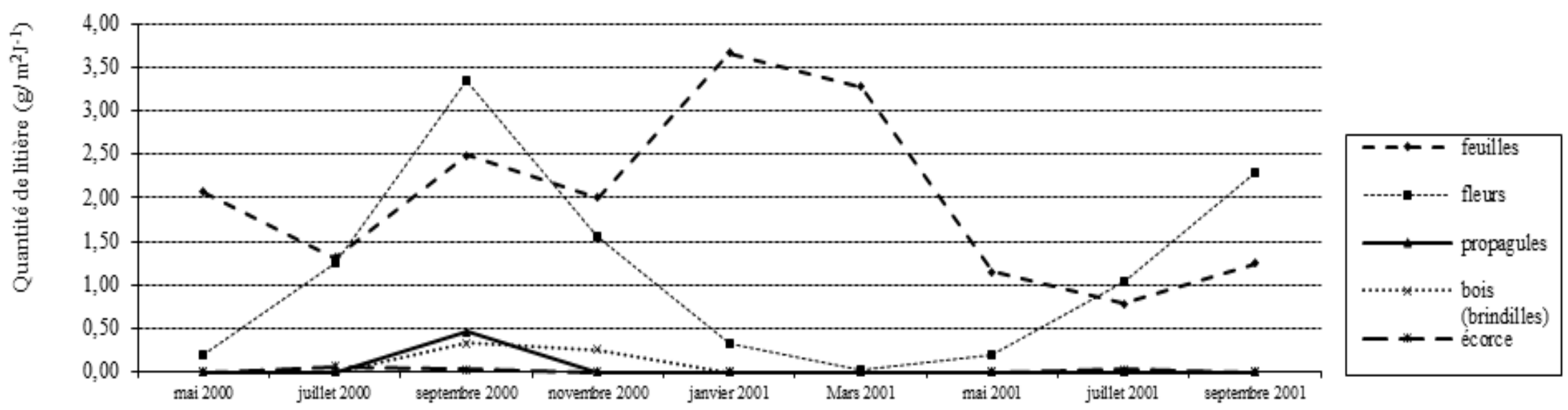

Figure 7a : Variation de la quantité de litière produite par le peuplement de Rhizophora sp. dans la trappe 1. 


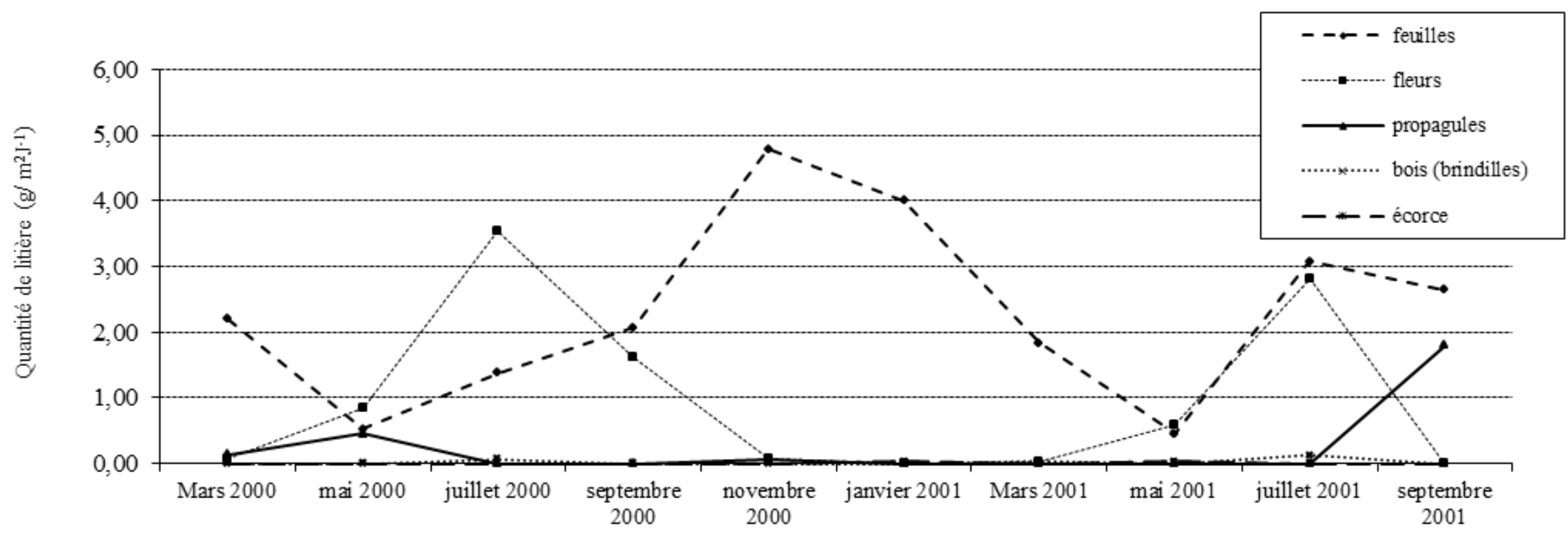

Figure 7b : Variation de la quantité de litière produite par le peuplement de Rhizophora racemosa/harisonnii dans la trappe 2. 
N. NDOUR et al. / Int. J. Biol. Chem. Sci. 7(6): 2349-2369, 2013

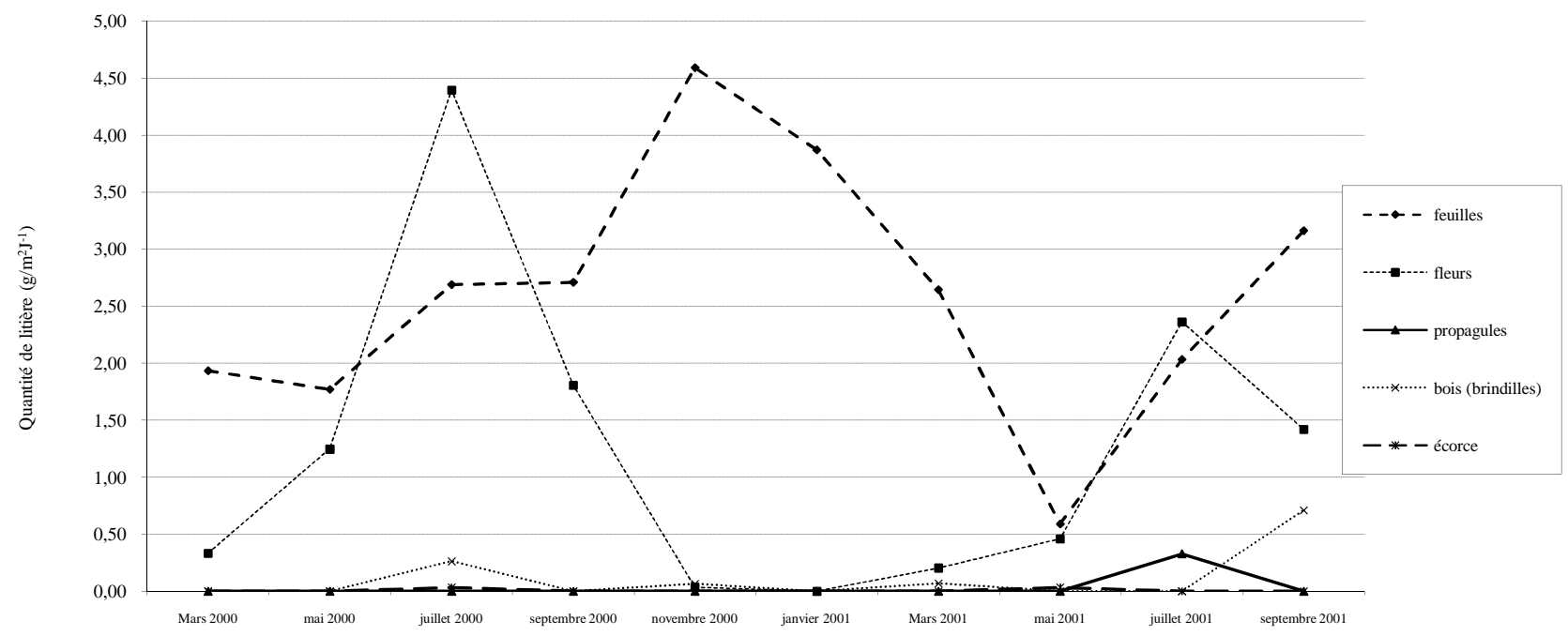

Figure 7c : Variation de la quantité de litière produite par le peuplement de Rhizophora sp. dans la trappe 3

Figure 7c : Variation de la quantité de litière produite par le peuplement de Rhizophora sp. dans la trappe 3. 
N. NDOUR et al. / Int. J. Biol. Chem. Sci. 7(6): 2349-2369, 2013

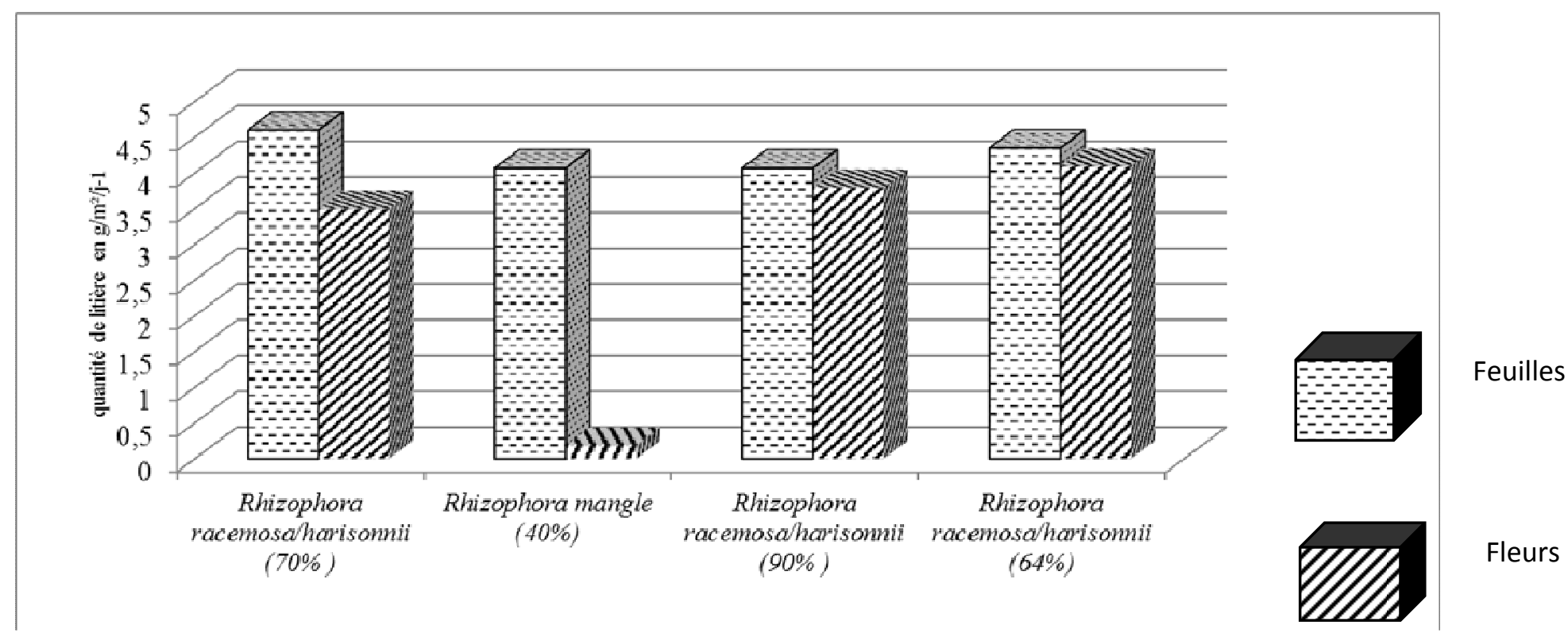

Figure 8 : Comparaison entre la production litière et le taux de recouvrement chez Rhizophora sp. 


\section{DISCUSSION}

L'étude montre que les forêts de mangrove sont sempervirentes et produisent continuellement de la matière organique en quantité importante dans leur milieu de vie. Cette continuité est liée à la diversité des éléments constitutifs de la litière (feuilles, fleurs et propagules) et à leur chute qui n'a pas lieu au même moment dans l'année. Cette situation est en adéquation avec la durée de vie des feuilles de $R$. racemosa/harisonnii estimée entre 6 à 12 mois par Gill et Tomlinson cités par Blasco (1987). La production de litière varie essentiellement entre 0,5 et $5 \mathrm{~g} / \mathrm{m}^{2} / \mathrm{J}$ pour les feuilles, entre 0 et $4,5 \mathrm{~g} / \mathrm{m}^{2} / \mathrm{J}$ pour les fleurs, et entre 0 et 0,5 $\mathrm{g} / \mathrm{m}^{2} / \mathrm{J}$ pour les propagules avec exceptionnellement un pic de $3 \mathrm{~g} / \mathrm{m}^{2} / \mathrm{J}$. L'estimation annuelle de cette production de litière dans le PNDS montre que 744,6 $\mathrm{g} / \mathrm{m}^{2} / \mathrm{an}$ de feuilles sont produits par les peuplements de Rhizophora mangle de taille moyenne, $1022 \mathrm{~g} / \mathrm{m}^{2} / \mathrm{an}$ par les strates hautes de Rhizophora racemosa/harisonnii et entre 708,1 et $934 \mathrm{~g} / \mathrm{m}^{2} / \mathrm{an}$ par les peuplements de taille moyenne de la même espèce. Ces chiffres montrent que la production foliaire est de loin la plus importante dans tous les deux sites étudiés. Ce résultat est en phase avec les conclusions de Menezes et al. (2008) qui démontrent que dans l'ensemble des études menées en Amazonie au Brésil, la quantité de feuilles qui chutent de la mangrove est toujours la plus importante par rapport aux autres composantes de la litière. Ces données confirment aussi l'importance de l'apport de la matière organique venant des peuplements de mangrove. Comparée à la mangrove du Mexique où Lugo et Snedaker (1974) estimaient la production foliaire de Rhizophora mangle à $896 \mathrm{~g} / \mathrm{m}^{2} / \mathrm{an}$, celle du PNDS est moins productive. Par contre, au Puerto Rico les peuplements de la même espèce produisent moins $\left(307 \mathrm{~g} / \mathrm{m}^{2} / \mathrm{an}\right)$ d'après Golley et al. cités par Blasco (1987). En Thaillande, Rhozophora apiculata BL. produit $2000 \mathrm{~g} / \mathrm{m}^{2} / \mathrm{an}$ selon Christensen cité par Blasco (1987). Il apparaît que la mangrove du PNDS est moins productive que celle de Thaillande alors que la mangrove à $R$. stylosa Griff. de l'île Okinawa au Japon qui produit (937 à 1321,9 g/m²/an) selon Sahadev et al. (2010) reste dans les mêmes proportions que celles de notre zone d'étude. Par ailleurs, ce travail a permis de montrer que les productions maximales de feuilles sont notées en novembre, mars, juillet et septembre pour $R$. racemosa/harisonnii et en juillet pour Rhizophora mangle. Selon Ellison (1997), les peuplements de Rhizophora mangle des Caraïbes perdent le maximum de feuilles entre mai et septembre pendant la saison des pluies ou des vents forts. La période de défoliation de l'espèce aux Caraïbes est la même qu'au PNDS. Cependant, la période de défoliation de $R$. racemosa/harisonnii qui va de janvier à septembre est plus longue que celle de $R$. mangle au Sénégal et aux Caraïbes. Il est à souligner aussi que la défoliation des espèces du genre Rhizophora est maximale pendant la saison des pluies au Saloum. C'est le cas de la mangrove du Mexique où les pertes maximales de litière surviennent pendant la saison des pluies (Flores-verdugo et al., 1997). Bien que la distance qui sépare le Sénégal (Afrique) et le Mexique (Amérique latine) soit grande, la phénophase foliaire de $R$. mangle reste similaire dans les deux pays. Des conditions pédoclimatiques, semblables dans les deux pays, pourraient en partie être l'une des raisons explicatives. Entre autres, cette étude a aussi permis de comparer la période de chute des fleurs du genre Rhizophora aux Caraïbes à celle du même genre au PNDS. Selon Ellison (1997), la chute des fleurs a lieu, aux Caraïbes, entre les mois d'août et de novembre avec un maximum en septembre. Au PNDS, la période de chute maximale des fleurs du genre Rhizophora est plus longue qu'aux Caraïbes. En référence aux travaux de Faye (2002), la floraison de l'espèce s'opère dans la période décembre-octobre. Cette période est quasi-semblable à celle identifiée par la présente étude. Considérant la quantité 
de fleurs par inflorescence et par espèce, $R$. racemosa/harisonnii (4 à 68 fleurs) produit largement plus que Rhizophora mangle (2 à 4 boutons floraux). Cependant, si l'on considère la quantité produite de propagules matures, c'est $R$. mangle, à inflorescence peu fournie, qui produit plus. Ce résultat est lié au taux d'avortement des fleurs qui est très élevé chez R. racemosa/harisonnii. Cette situation laisse penser que $R$. mangle risque dans le long terme d'occuper l'espace au détriment de $R$. racemosa/harisonnii. Cette hypothèse pourrait trouver aussi sa raison d'être dans la coupe de bois de palétuviers qui, sans distinction d'espèces, peut contribuer à la perturbation du cycle végétatif des palétuviers et de leur productivité. Inversement, l'étude a fait également ressortir la faible production de semences de $R$. racemosa/harosonnii par rapport à la quantité de fleurs par inflorescence. Cette situation est-elle normale ou est-ce alors une conséquence des effets de la sécheresse, d'un problème de pollinisation ou d'agents pollinisateurs ou d'autres explications? Il urge que les botanistes se penchent sur cette question préoccupante qui risque de changer les rapports d'occupation de l'espace par les palétuviers au Delta du Saloum. Les résultats de l'étude font également remarquer que la production foliaire est plus faible au moment où la production florale démarre ou atteint son pic le plus élevé. Le démarrage de la floraison de Rhizophora mangle correspond généralement au pic de production des propagules de deuxième génération chez $R$. racemosa/harisonnii. Le pic de floraison de cette dernière coïncide, par contre, avec la prolifération des propagules de $R$. mangle. Par ailleurs, le pic de production de propagules, le plus élevé, a lieu entre les mois de juillet et septembre pour Rhizophora mangle. La saison des pluies et les vents qui l'accompagnent peuvent expliquer la chute importante des propagules matures ou immatures dans les trappes.
Le bois produit est constitué de petites branches, de brindilles et de débris de bois mort. Les effets des vents de la saison des pluies expliquent en partie l'augmentation des quantités de bois au cours de cette période. Toute la litière qui chute participe à l'enrichissement du milieu par accumulation, biodégradation et transformation de la matière organique par les décomposeurs. Après le recyclage de la matière organique, les sels minéraux issus de la litière sont réutilisés en partie par les palétuviers et le phytoplancton (FAO, 1994 ; Faye et al., 2008). La litière mérite donc une attention particulière d'autant plus qu'elle représente un maillon important de la chaîne alimentaire. De plus, la litière transformée en sel minéraux permet à la mangrove d'échanger des éléments avec les écosystèmes qui lui sont contigus. Cet échange est important pour les autres écosystèmes, particulièrement l'écosystème marin où l'on note la présence de forêts sousmarines et d'herbiers marins constitués essentiellement d'algues (Carter et al., 1973 ; Pool et al., 1975 cités par la FAO, 1994). La présente étude a permis aussi de montrer qu'il n'y a pas de corrélations entre la production litière, la taille du peuplement et son taux de recouvrement au sein de la même espèce. Par contre, lorsque la composition floristique est différente, la litière florale est plus importante chez $R$. racemosa/harisonnii. Cependant, Faye et al. (2008) ont montré qu'il existe des corrélations entre la biomasse foliaire et les facteurs édaphiques. L'abondance de certains éléments chimiques du sol expliquerait un bon développement végétatif chez les palétuviers et une bonne production de biomasse foliaire.

\section{REMERCIEMENTS}

Ce travail de recherche a été réalisé sur la base d'un programme de «recherche expérimentale sur les techniques de gestion des forêts de mangrove» co-piloté par la Faculté des Sciences Agronomiques de Gembloux (FUSAGx) et la Direction des Parcs Nationaux (DPN) du Sénégal. La 
collecte des données et leur analyse a été confiée à l'Institut des Sciences de l'Environnement de l'Université Cheikh Anta Diop de Dakar qui remercie vivement l'ensemble des institutions suscitées, divers acteurs au développement, les populations locales du Delta du Saloum et tous ceux qui ont contribué à l'amélioration de ce document.

\section{REFERENCES}

Bertrand F. 1993. Contribution à l'étude de l'Environnement et de la dynamique des mangroves de Guinée. Données de terrain et apport de télédétection, Paris, ORSTOM, coll. Etudes et Thèse de doctorat, Université de Bordeaux III, UER de géographie, 201p.

Blasco F. 1984. Taxonomic considerations of the mangrove species. In the Mangrove Ecosystem: Research Methods. UNESCO/SCOR: New Delhi ; 81-90.

Blasco F, Achard F. 1987. Productivité primaire dans les mangroves Approches quantitatives et qualitatives. COMARAF, PNUD - UNESCO, Série documentaire 1 : Paris ; 27.

Brown MS. 1984. Mangrove litter production and dynamics. In the Mangrove Ecosystem: Research Methods, Snedaker SC, Snedaker JG (eds) ; 231-237.

Dacosta H, Ndiaye A, Diouf PS. 1998. Inventaire des zones humides du Delta $\mathrm{du}$ Saloum. Rapport de consultation, Dakar, 34 p.

Diaw AT. 1997. Evolution des milieux littoraux du Sénégal. Géomorphologie et Télédétection. Note bibliographiques, Thèse de Doctorat d'Etat ès Lettres, Université de Paris I PanthéonSorbonne, 267p.

Diouf PS. 1996. Les peuplements de poissons des milieux estuariens de l'Afrique de l'Ouest : l'exemple de l'estuaire hyperhalin du Sine Saloum, Thèse de doctorat, Université de Montpellier II, 267 p.
Ellison JC. 1997. Mangrove community characteristics and litter production in Bermuda. In Mangrove Ecosystem Studies in Latin America and Africa, Björn, Kjerfve, Luiz Drude de Lacerda, El Hadji Salif Diop (eds); 9-17.

FAO. 1994. Les relations trophiques dans les écosystèmes de mangrove. Extrait de "mangrove forest management guidelines". FAO Forestry Paper No. 117, Rome.

Faye MN. 2002. Etude phénologique du genre Rhizophora et influences des facteurs édaphologiques ( $\mathrm{pH}$ et salinité de l'eau) sur la végétation de mangrove de l'estuaire du Saloum, Sénégal. Mémoire de DEA, UCAD, 42p + annexes.

Faye MN, Kaly JL, Diallo A, Guissé A. 2008. Influence des facteurs édaphiques sur la biomasse foliaire de deux espèces de palétuviers (Rhizophora sp., Avicennia africana) dans la Réserve de Biosphère du Delta du Saloum, Sénégal. Journal des Sciences et Technologies, 7: 35-42.

Flores-Verdgo FJ, Gonzàles-Farias M, Blanco-Correa, Nuñez-Pastén A. 1997. The Teacapan-Agua Brava-Marimas Nationales mangrove ecosystem on the Pacifique cost of Mexico. In Mangrove Ecosystem Studies in Latin America and Africa, Björn, K, Luiz Drude de L, El Hadji Salif Diop (eds); 35-46.

Lugo AE, Snedaker SC. 1974. The ecology of mangrove. Ann. Rev. Ecol.syst., 5: 3964.

Marius C. 1984. Contribution à l'Etude des Mangroves du Sénégal et de la GambieEcologie-Pédologie-Géochimie. Mise en Valeur et Aménagement. ORSTOM, Paris, 309 p.

Menezes MP, Berger U, Mehlig U. 2008. Mangrove vegetation in Amazonia: a review of studies from the coast of Para and Maranhao States, north Brazil. Acta Amazonica, 38(3): 403-420.

Ndour N. 2005. Caractérisation et étude de la dynamique des peuplements de 
mangrove du Delta du Saloum (République du Sénégal). Dakar, Faculté des Sciences et techniques, Thèse de troisième cycle, Université Cheikh Anta Diop, 180 p.

Ndour N, Dieng DS, Fall M. 2012. «Rôles des mangroves, modes et perspectives de gestion au Delta du Saloum (Sénégal)». Rev VertigO, 11(3): 16p.

Sahadev S, Rafiqul Hoque ATM, Analuddin K, Hagihara A. 2010. Phenology and litterfall production of mangrove Rhizophora stylosa Griff. in the subtropical region, Okinawa Island, Japan; proc. of international Conférence Aspects of Bangladesh (ICEAB10), Japan, pp87-90.

Soumaré A. $1992 . \quad$ Evolution géomorphologique récente des paysages du Bas-Saloum. Dakar, Faculté des lettres et sciences humaines, Mémoire de DEA, Université Cheikh Anta Diop; $61 p$.

Rajendran N, Kathiresan K. 2007. Microbial flora associated with submerged mangrove leaf litter in India. Rev. Biol. Trop., 555 (2): 393-400.

Thomas YF, Diaw AT. 1997. Suivi (1984 1993) de la rupture de la flèche de Sangomar, estuaire du fleuve Saloum, Sénégal. Photo-Interprétation, 3-4 : 199204.

Throchain JL. 1940. Contribution à l'étude de la végétation du Sénégal. Mémoire de Doctorat de L'Université Bordeaux 3, $433 \mathrm{p}$. 